\title{
An important challenge in occupational health: sampling indoor airborne viruses
}

\author{
Diana M. Cocârță ${ }^{1 *}$, Cristina A. Gogoncea ${ }^{1}$, and Constantin Streche $^{1 \dagger}$ \\ ${ }^{1}$ University POLITEHNICA of Bucharest, Faculty of Power Engineering, Department of Energy \\ Production and Use, RO-060042 Bucharest, Romania
}

\begin{abstract}
Airborne biological particles are made by bacteria, viruses, fungi, pollen, dust mites and other living organisms. Between the airborne biological contaminants, the sampling of airborne viruses is the most difficult. This technical challenge has real consequences both in limiting the studies on the aerobiology of viral diseases, and in making possible, at least for now, to establish Standards for Acceptable Bioaerosol Exposure Limits in case of viruses. The circumstances and uncertainties in which we are living from the end of 2019 , have demonstrated once again, the need of having common approaches in sampling, processing, and analysis of bioaerosol samples. Results from sampling indoor airborne viruses can be used both for identifying the airborne transmission of the respiratory viruses and for providing useful information as support in human health risk assessments. On the other hand, even if there are different sampling methods and equipment used for collecting bioaerosols, there are certain elements that must be considered in choosing the most appropriate sampling method to collect airborne viruses. The main challenge in sampling indoor airborne viruses is to identify the system that allows the sampling of bioaerosols ensuring in the same time the viability of the virus and the representativeness of the sample. Keeping in mind the lack of standardization in sampling indoor airborne viruses, as well as the missing of common approaches for air sampling strategies or sample treatment and analysis, the present work is focused on providing important recommendations as support in the concentrated effort of the researchers in developing of a common strategy for indoor viable bioaerosol sampling for virus detection.
\end{abstract}

\section{Introduction}

Bioaerosols containing biological material can include different components as bacterial cells and cellular fragments, fungi, pollens, viruses, allergens, by-products of microbial metabolism and others [1-3]. Biological aerosols could be generated both in outdoor and indoor environments [1] [3] having as origin different natural and anthropogenic sources, humans themselves being the most important aerosol source. These can be divided in viable bioaerosols (e.g. bacteria, fungi, viruses) and non-viable bioaerosols (e.g. endotoxins and

\footnotetext{
* Corresponding author: dianacocarta13@yahoo.com

${ }^{\dagger}$ Corresponding author: constantin streche@yahoo.com
} 
proteins) [4]. According to World Health Organisation (WHO) [5], exposure to airborne biological contaminants has a real contribution in increasing population morbidity and mortality. Important health effects because of exposure to bio-aerosols are already known when existing in high concentrations in indoor environments [3] [6]. Moreover, circumstances and uncertainties in which we are living from the end of 2019 generated by the pandemic crisis, have demonstrated once again, the need of having common approaches in sampling, processing and analysis of bioaerosol samples with the aim of identifying the transmission of airborne respiratory viruses. Even if this necessity was underlined by different authors also before the Severe Acute Respiratory Syndrome COronaVirus 2 (SARSCoV-2) crisis [7-9], now became an emerging priority for the public authorities and, consequently, for the scientists. So, at international level it is really evidenced an increased focus on the importance of bioaerosols.

Between the airborne biological contaminants, the sampling of airborne viruses is the most difficult. This difficulty is given by their structure (DNA or RNA genomes) that is enclosed in a fatty liquid membrane. This is a consequence of the fact that viruses need of a host cell to reproduce and for inducing the infection [10]. This technical challenge has real consequences both in limiting the studies on the aerobiology of viral diseases [11], and in making possible for now to establish Standards for Acceptable Bioaerosol Exposure Limits in case of viruses ("exposure limits for airborne fungi and bacteria are limited, while standards for viruses do not exist") [11]. Moreover, as Mbareche is underlying in a recent research work [7], another limitation is given by the fact that, the diversity of the approaches used even from the sampling phase of the airborne viruses, it makes that, results obtained from studies that initially have the same purpose, cannot be compared with each other.

The main challenge in sampling indoor airborne viruses remains the identification of the system that allows the sampling of bioaerosols ensuring in the same time the viability of the virus and the representativeness of the sample [7] [12] [14]. Keeping in mind the lack of standardization in sampling indoor airborne viruses, as well as the missing of common approaches for air sampling strategies or sample treatment and analysis, the present work is focused on providing important recommendations and remarks as support in the concentrated effort of the researchers in developing of a common strategy for sampling viruses in air. Medical personnel exposure to viruses is an actual and significant issue that can be well done only when supported by appropriate solutions for sampling of the airborne viruses. Only in this way, the assessment of occupational exposure of medical personnel could be ensured in appropriate and accurate conditions.

\section{Methods and samplers used to collect airborne viruses}

Important reasons for which at the beginning of the pandemic crisis there was a disagreement of the experts [15] and public health officials (WHO) [16] about airborne transmission of SARS-CoV-2 are because the preliminary studies were evidencing contradictory results. While the WHO on March 27, 2020 was indicating that "there is not enough evidence to suggest that SARS-CoV-2 is airborne" [16], different other studies were providing contrary results [17-20]. In this context, it is important to be mentioned that, the diversity of the approaches used even from the sampling phase of the airborne viruses, it makes that, results obtained from studies that initially have the same purpose, cannot be compared with each other [7]. On the other hand, even if there is an important representativeness of the studies on monitoring airborne bacteria in indoor and outdoor environments [21], there is a limited number of studies evaluating the presence of airborne viruses correlated with their infectious potential [22]. Additionally, common standards, methods or protocols for sampling and determination of airborne viruses are not existing [11]. 
Currently, methods used for collecting and analysing airborne viruses are usually divided in culture-based and culture-independent methods [11] [14] [21-23]. The first category is assuming that the virus viability is maintained during and after bioaerosol collection and are commonly used to estimate airborne virus exposures in indoor environments, presuming the cultivation of microorganisms from the environmental sample [9]. Culture-independent methods, as expected, don't need microbial cultivation and are involving the analysis of nucleic acids extracted from the sample [23]. On the other hand, the selection of the sampler type used for sampling viruses, from air, as well as sampling conditions, are still important challenges in bioaerosol science.

In the framework of the second part of the present study, the attention is paid to different methods that were already used for sampling airborne microorganisms, particularly for sampling aerosols with the aim of virus's detection.

\subsection{Impactors and cyclones}

Impactors are simple devices used since 1800s for studying relationship between particle pollution and diseases that are involve the existence of a sequence of nozzles and a surface where the impaction is taking place [24]. These kinds of devices are sampling both solid and imperceptible particles that are floating in a gas (in air) based on the tendency of particles to move in a straight-line motion, due to their inertia, when an obstacle appears in the way of the airflow [25]. Particles present in the air that have a high inertia (high mass of the particle) will tend to keep the fluid direction (moving in the direction of the initial airflow) when there is an unexpected change of the airflow direction; thus, they will leave the direction imposed by the impactor construction (they will come out of the airflow) and they will impact the collection plate (impaction plate). Similarly, particles smaller than the particles retained in the first stage of a cascading impactor will follow the airflow and will end up impacting the collection plates of the lower steps which have smaller and smaller nozzles ensuring the passages between the impactor stages. Accordingly, the particles present in the airflow can adhere to the impact plates and can accumulate on these surfaces, the impactors becoming devices that are used for the sampling of particles with dimensions between $0.05-30 \mu \mathrm{m}$ [25].

The efficiency of the impactors $(\eta)$ for the sampled particles depends directly on the particle diameter (D), the gas flow rate (V) and the density of the particle that was sampled $(\rho)$; varies inversely with the gas viscosity $(\mu)$ and with a parameter $\left(D_{b}\right)$, characterized by the impactor dimensions (e.g. the diameter of the inlet nozzle), which is related to the curvature of the air flow [27].

$$
\eta=D 2 V \rho / 18 \mu D_{b}
$$

The open access literature is evidencing that the highest impaction efficiency is ensured by large and dense particles, and high flow velocities [27]. Well-known impactors are the Andersen [27] and Casella slit samplers [28 - 29], that were already used for sampling viruses on large particles [13] [30].

Liquid cyclonic collectors are also used for collecting airborne viruses, not being appropriate for small-sized virus-containing particles, existing in the same time the risk of viruses deactivating because of the physical damage of the sample. In case of these samplers, the liquid medium moves away and subsequently, the particles trapped against the cyclone's wall are collected [13]. 


\subsection{Liquid impingers}

The liquid impingers are another category of devices used for sampling of airborne viruses [31], demonstrating their high collection efficiency for particles $>0.1 \mu \mathrm{m}$ [32]. These sampling systems consist in small bottles coupled with an air sample pump to ensure a known volume of air. Thus, airborne viruses are transferred into collection liquids for further analysis. The main advantage of using liquid impingers for sampling the viral particles is the fact that the liquid media is ensuring the moisture conditions of the biological and, in this way, a correct subsequent analysis can be done [31].

\subsection{Filters}

Mechanisms as electrostatic attraction, interception, diffusion, inertial impaction and gravitational settling are influencing the filters efficiency in sampling airborne viruses [33]. Filters commonly used in sampling airborne viruses are made of cotton, cellulose, polytetrafluoroethylene (PTFE) and polycarbonate (PCTE), according to the target viruses' type [11]. Even if generally filters are not considered as the first option in order to sample airborne viruses, it seems that gelatin filters are most appropriate for the collection of airborne viruses. This option does not significantly affect the virus infectivity, but there are different other limitations because of the environmental conditions (e.g. both low and high humidity can cause the damage of the sample) [11]. Currently, filters were used by the Italian researchers for evidencing that SARS-CoV-2 RNA can be identified on particulate matter $(\mathrm{PM})$, suggesting in this way a possible approach to be used as indicator of epidemic recurrence [34].

\subsection{Condensation-based growth tube collectors}

Growth tube collector (GTC) that uses the laminar-flow water-based condensation to facilitate particle growth [35] is another sampling method for collecting airborne viruses. This sampling solution is allowing the collection of bioaerosols with a diameter $<10 \mathrm{~nm}$ up to $10 \mu \mathrm{m}$; the fine particles are gentle sampled into a liquid medium through condensationenhanced inertial deposition. This solution is simulating the behaviour of the human lungs in a cold day; cold aerosol particles are introduced into a warm GTC saturated with water vapour [36]. Experimental research involving this approach showed that GTC is an important option for the investigation of infectious virus particle demonstrating an efficiency for the collection of airborne viruses seven times higher than that of the BioSampler [37]. The BioSampler is working as a glass liquid impinger, having a curved inlet tube and a vacuum in the flask to force the air through the sampler. It has with three tangential sonic nozzles, forcing the air at sonic speed through these nozzles. During the sampling, the collection liquid existing in the flask is moving in a swirling motion [11].

As previously illustrated, there are numerous sampling devices and approaches that can be used with the aim of detecting airborne viruses. Establishing the purpose of the sampling is the starting point in in choosing the appropriate solution. On the other, choosing the right sampling method that could ensure both physical and biological integrity of the sample must consider also other factors as relative humidity $(\mathrm{RH})$, temperature, radiation, chemical composition of the air (environmental factors), as well as aerosolization medium and exposure period [37]. 


\section{Conclusions and remarks}

The current pandemic of coronavirus disease 2019 (COVID-19) caused by spread of SARSCoV-2 have increased the global concerns about the airborne transmission of infectious viruses. Mitigation measures, as well as proper precautionary actions can be applied if the knowledge on hazard posed by the airborne viruses is improved and well understood.

The main challenge in sampling indoor airborne viruses is to identify the system that allows the sampling of bioaerosols ensuring in the same time the viability of the virus and the representativeness of the sample. The viability of the virus is ensured by a proper efficiency for viral recovery of the sampler (in other words it is about the biological efficiency), while the physical efficiency is guaranteed by a proper rate of recovery of different particle sizes.

Specific information illustrated within the present study, leads to different significant reflections that may be considered for future research that needs for improved solutions and approches to better understand indoor bioaerosols:

- Regarding the sampling of airborne viruses in the air of workplaces, currently, no common approach/protocol has been suggested by the organizations for the sampling and determination of viruses in the air. This is a consequence of the fact that, each virus, according to its structural composition and interactions with other aerosol components, reacts in a specific way to each factor (e.g. environmental factors, aerosolization medium or exposure period).

- Information presented in the current paper is supporting the development of our capacity for a better understanding of different methods for sampling airborne viruses, considering for each proposed solution its particularities.

- Virus particles may be sampled from air by considering different approaches and sampling strategies but in choosing one of them, we must be guided by the biological and physical efficiency of the proposed solution, and by the final objective of the exposure scenario evaluation.

- In the framework of the current pandemic crisis, more specific research concerning SARS-CoV-2 infection transfer mechanisms should be done in case of people interacting in indoor environments.

- Effectively sizing the particles with infectious viruses could provide a better understanding of the aerosol behaviour with important consequences in the spread of the infection by aerosolised particles.

- Biological hazards due to airborne viruses' exposures in occupational environments will be better understood if we will be able to identify appropriate solutions for ensuring the integrity and infectivity of the sampled viruses.

- The analytical methods used for post-sampling analysis, as well as viruses' type are driving the selection of the bioaerosol collection methods.

Due the fact that previously research studies focused on detecting airborne viruses have used different approaches and devices with different parameters for sampling (e.g. sampling time and flow rates), as well as a variety of places used for studying the airborne transmission of an infectious disease (e.g. schools, hospitals, dental clinics and others), the achieved results were not consistent results. In this concern, future research needs to focus on both methods to better understand indoor bioaerosols aiming and common approaches for sampling, processing, and analysis of bioaerosol samples to identify the airborne transmission of the respiratory viruses.

Based on information generated by the application of common approaches used in studying indoor bioaerosols, public authorities will be certainly supported in taking the best 
decisions for establishing the public health policies and recommendations, becoming able to provide active control of the pandemics and to ensure healthy workplace environments.

This work was supported by the Erasmus + Programme, SafeEngine project, contract no 2020-1-RO01KA203-080085.

\section{References}

1. L.D. Stetzenbach, Moselio S (ed) Encyclopedia of Microbiology, 3rd edn. Academic Press, Oxford, p 175-182 (2009)

2. B. Ghosh, H. Lal, A. Srivastava, Environ Int. 85, 254 (2015)

3. P. Srikanth, S. Sudharsanam, R. Steinberg, Indian J Med Microbiol 26, 302 (2008)

4. U. Turaga, R.J. Kendall, V. Singh, M. Lalagiri, S.S. Ramkumar, E. Sparks (Ed.), Advances in Military Textiles and Personal Equipment, (Woodhead Publishing, USA, 2012)

5. World Health Organization, Indoor air quality: Biological contaminants, (WHO regional publication, Denmark, 1988)

6. R.I. Moraru, G.B. Băbuț, L.I. Cioca, Environ Eng Manag J 13, 6 (2014)

7. H. Mbareche, L. Morawaska, C. Duchaine, J. Air Waste Manag. Assoc., 69, 789 (2019)

8. K.H. Kim, E. Kabir, S.A. Jahan, J Environ Sci (China) 67, 23 (2018)

9. J. Cox, H. Mbareche, W.G. Lindsley, C. Duchaine, Aerosol Sci. Technol., 54, 5572 (2020)

10. A.R. Rahmani, M. Leili, G. Azarian, A. Poormohammadi, Sci Tot Env, 740 (2020)

11. D. Verreault, S. Moineau, C, Duchaine, Microbiol Mol Biol Rev 72, 413 (2008)

12. C. J. Hogan, E.M. Kettleson, M-H Lee, B. Ramaswami, L.T. Angenent, P. Biswas, J. Appl. Microbiol. 99, 61422 (2005)

13. M. Pan, J.A. Lednicky, C.Y. Wu, J Appl Microbiol, 127, 61596 (2019)

14. P. Duquenne, Annals of Work Exposures and Health, 62, 2139 (2018)

15. Lewis D. Is the coronavirus airborne? Experts can't agree. Nature, 580, 7802 (2020)

16. https://www.who.int/news-room/commentaries/detail/modes-of-transmission-of-viruscausing-covid-19-implications-for-ipc-precaution-recommendations 12.03.2021]

17. Y. Liu, Z. Ning, Y. Chen, M. Guo, Y. Liu, N.K. Gali, bioRxiv (2020) (preprint)

18. S.W.X. Ong, Y.K. Tan, P.Y. Chia, et al. JAMA, 323, 16, 1610 (2020)

19. van Doremalen, N. et al., N. Engl. J. Med. 382, 1564 (2020)

20. L. Setti, F. Passarini, G. De Gennaro, P. Barbieri, M.G. Perrone, M. Borelli, J. Palmisani, A. Di Gilio, P. Piscitelli, A. Miani, Int. J. Environ. Res. Public Health 17, 8, 2932 (2020)

21. K.K. Coleman, T.T. Nguyen, S. Yadana, C. Hansen-Estruch, W.G. Lindsley, G.C. Gray, Sci. Rep., 8 1-7 (2018)

22. E. Franchitti, E. Pascale, E. Fea, E. Anedda, D. Traversi, Atmosphere, 11, 452 (2020)

23. W.G. Lindsley, B.J. Green, F.M. Blachere, S.B. Martin, B.F. Law, P.A. Jensen, M.P. Schafer, in NIOSH Manual of Analytical Methods (National Institute for Occupational Safety and Health, Cincinnati, $\mathrm{OH}, 2017$ )

24. V.A. Marple, Aerosol Science and Technology, 38 3, 247 (2004)

25. B.J. Finlayson-Pitts, J.N. Pitts, Chemistry of the Upper and Lower Atmosphere, Academic Press, USA, 1999)

26. A.A. Andersen, J Bacteriol, 76: 357 (1958)

27. W. John, Aerosol Science and Technology, 23, 1, 2 (1995)

28. W. Whyte, Journal of Hospital Infection, 2, 297 (1981)

29. A Carducci, I. Federigi, M. Verani, Atmosphere 11, 7710 (2020) 
30. G. Song, T.H. Kuehn, M. Abin, H. Verma, A. Bekele, S.K. Mor, S.M. Goyal, J. Appert, P.C. Raynor, Z. Zuo, Aerosol Science and Technology, 48, 12, 1360 (2014)

31. Y.C. Chen, I.J. Wang, C.C. Cheng et al., Aerobiologia 385 (2021)

32. A. Dart, J. Thornburg, Atmospheric Environment, 42, 4, 828 (2008)

33. W.C. Hinds, Aerosol technology, 2nd edition, (Wiley-Interscience, NY, 1999)

34. L. Setti, F. Passarini, G. De Gennaro, P. Barbieri, M.G. Perrone, M. Borelli, J. Palmisani, A. Di Gilio, V. Torboli, F. Fontana, L. Clemente, A. Pallavicini, M. Ruscio, P. Piscitelli, A. Miani, Environmental Research, 188, 109754 (2020)

35. M. Pan, A. Eiguren-Fernandez, H. Hsieh, N. Afshar-Mohajer, S.V. Hering, J. Lednicky, Z. Hugh Fan, C.-Y. Wu, Journal of Applied Microbiology (2015)

36. W.G. Lindsley, B.J. Green, F.M. Blachere, et al., NIOSH Manual of Analytical Methods (NMAM), 5th Edition (NIOSH, USA, 2017)

37. J. Lednicky, M.H. Pan, J. Loeb, H. Hsieh, A. Eiguren-Fernandez, S. Hering, H.Z. Fan, C.Y. Wu, Aerosol Sci Technol, 50, I-IV (2016) 